Editorials

\title{
What You See Is What You Get
}

\author{
${ }^{1}$ Saadettin Sel, ${ }^{2}$ Mark A. Brown, ${ }^{3}$ Joachim Storsberg and ${ }^{3,4}$ Christian Schmidt \\ ${ }^{1}$ Department of Ophthalmology, University of Heidelberg, Heidelberg, Germany \\ ${ }^{2}$ Department of Clinical Sciences and Colorado School of Public Health, \\ Colorado State University, Fort Collins, CO 80523-1052, USA \\ ${ }^{3}$ Department of Biomaterials and Healthcare, Division of Life Science and Bioprocesses, \\ Fraunhofer Institute for Applied Polymer Research (IAP), Geiselbergstraße 69, 14476 Potsdam-Golm, Germany \\ ${ }^{4}$ Editorial Office, The American Journal of Immunology, S-207, 244, 5th Avenue, \\ New York, NY, 10001 USA and S-71, 1A, 400, King William St, Adelaide, SA 5000, Australia
}

Article history

Received: 14-09-2016

Revised: 26-09-2016

Accepted: 28-09-2016

Corresponding Author: Christian Schmidt

Editorial Office, The American Journal of Immunology, S-207, 244, 5th Avenue, New York,

NY, 10001 USA and S-71, 1A, 400, King William St,

Adelaide, SA 5000, Australia

Email: schmidt102@gmail.com

\section{Saadettin Sel}

Department of Ophthalmology,

University of Heidelberg,

Heidelberg, Germany

Email: Sel@med.uni-heidelberg.de

\begin{abstract}
The recognition and neutralization of invading pathogens by the human immune system is designed to protect the organism. Sometimes, the checks and balances in place securing a tightly controlled immune surveillance of privileged sites are lifted in order to ensure survival of the individual at the cost of losing the functionality of an organ. Here, we use choice areas to argue that, while much has been learned, further studies are needed to explain the lingering enigma of privileged immunity.
\end{abstract}

Keywords: Immunity, Eye, Privilege, Surgery
The notion that local control at sites of origin and metastasis of cancerous lesions can be traced to early publications, such as Burnet (1957) and has not been contested so far. An extensive array of examples for a tightly regulated local control, or, as it is also referred to, the immunological surveillance and how this interplay is expropriated by malignant lesions is investigated by many groups.

As example in point, metastases to the bone can be correlated with the presence of soluble factors and cell types implicated in soothing the immune response on the one hand and a small number of what is termed cytotoxic cell types (Baschuk et al., 2015). Another example of this apparent compromise between local surveillance and sites of especially restricted supervision by the immune system is the eye. Here, cases are reported where survival of the organism is secured at the cost of lost vision (Niederkorn and Stein-Streilein, 2010).

What is the implication for the development of new materials designed to aid caring ophthalmologists? One easy answer is that the local immunological situation ought to be taken into account when new materials are to be developed. This, however, is easier said than done because most cases of patients where the caring ophthalmologist recommends the use of synthetic materials are accompanied by either a compromised immune system or an immunological defense ready to fight severe infections, some of them may even threaten the patient's survival. Looking at the progress made on the side of materials, e.g., Griffith and Harkin (2014), ophthalmologists find themselves often in a dilemma where they have to weigh risks of an intervention against the resulting benefits as they are the ones who have to advise a patient of the options available.

All this is compounded by deceptively simple questions, such as the appropriate suture material for corneal surgery. Documented records describing techniques to close wounds or incisions using surgical gown prepared from animal connective tissue can be dated back to at least 2,500 B.C. (McPherson, 1959). The enthusiasm of the use of resorbable material was curbed, however, by Halsted (1913) arguing that said materials are likely to elicit adverse reactions by the host. Instead, the author recommended the use of non- 
resorbable ligatures or sutures, such as silk-based materials (Halsted, 1913). The enthusiasm associated with the use of non-absorbable suture materials was dampened by observations from Salthouse and Matlaga (1975). The authors observed an apparently pathological cellular enzyme activity and presence of bone marrow originating macrophages at rat gluteal muscle implant sites where nonabsorbable materials, such as polyester, polypropylene and silk, were used. Furthermore, all materials used were encapsulated at day 21 (Salthouse and Matlaga, 1975).

Later investigators used Halsted's (1913) critique on absorbable suture materials to establish a study object to investigate the pathologic phenomenon. Using rabbits as a model organism, Salthouse et al. (1970) reported two phases in enzyme activity as absorbable material is degraded in corneal suture sites: Alkaline phosphatase in an early phase ( 3 to 14 days) with polymorphonuclear leucocytes present adjacent to the suture-tissue interface and a later phase with a marked acidic phosphatase activity (14 to 21 days) with round monocytes and keratinocytes present adjacent to the suture-tissue interface. Salthouse (1976) attributed the apparent alkaline phosphatase to an activity associated with keratinocytes and the observed acidic phosphatase to the activity of monocytes.

The notion that irritation of the cornea results in the appearance of capillaries and lymphatics, which may undergo atrophy, e.g., Lightbody (1867), is, so far, not contested in the literature. It must be noted that there appears to be no consensus in the literature when it comes to the issue of corneal vascularity, encompassing blood and lymphatic systems. As a result of examining healthy corneas from mammals and birds, the author described nucleated cells, resembling granular white blood cells, "occurring along one or both sides of the capillaries" (Lightbody, 1867). Notably, these cells were found to be "crowded and rounded towards the cornea [and] scattered towards the sclera" (Lightbody, 1867).

Studies of restricted corneal burns in rabbits revealed that lesions at a distance greater than a critical radius $(r>$ $4.2 \mathrm{~mm}$ ) from the limbus to the center of the cornea failed to yield a visible vascularization. Injuries inside the circular segment $(\mathrm{r}<4.2 \mathrm{~mm})$ from the limbus to the center of the cornea resulted in vessels growing from the limbus nearest to the trauma in the form of an isosceles triangle (Campbell and Michaelson, 1949). These studies would strongly suggest that either a removal of a repression or a de-novo synthesis of a secreted factor may be at the center of an injury-induced vascularization of rabbit corneas.

Later studies revealed that there is a VEGF "trap" in the mouse cornea (see below), based on genetic studies revealing that 2 out of 24 patients suffering from sporadic aniridia (Nelson et al., 1984) displayed mutations in the human homolog of the murine Pax-6 locus, both affecting protein function (Jordan et al., 1992). The subsequently generated mouse model (Ramaesh et al., 2006) was then used to investigate the abnormal vascularization of the cornea of Pax-6 heterozygous deficient (Pax-6+/-) mice by Ambati et al. (2006). The authors traced this phenomenon to the absence of the endogenous VEGF-1 "trap", namely absence of the soluble form of the VRGF-1 receptor (sFLT-1; Ambati et al., 2006; Kendall et al., 1996). Significantly, the authors could confirm these observations using immunohistochemistry on a cornea of a patient with aniridia-associated vascularization (Figure 4D in Ambati et al. (2006)). Further support for this notion of suppression of angiogenesis in mouse models comes from Cursiefen et al. (2006a) where the surface of murine corneal epithelium is found to be decorated with non-vascular VEGF receptor 3 and linked to a suppression of inflammation-elicited corneal angiogenesis. Taken together, it appears that there may be another complex regulation network involved in the suppression of corneal angiogenesis in the murine cornea.

As justly pointed out by Cursiefen et al. (2006a) and Ambati et al. (2006), any generalization of their findings, in terms of including but not limited to the redundant regulation of vascularization, the immunological privilege of the cornea and the extrapolation to other species beyond their models tested, ought to be supported by formal experimental evidence. Whilst this should not and cannot be construed as an invalidity of murine models of certain maladies for basic immunological science, "[...] there is a serious problem, which is that virtually none of the advances in basic immunology [...] have been incorporated into standard medical practice [...]" (Davis, 2008). Credence to Davis' concern is provided by Seok et al. (2013) where the response of mice and humans to acute inflammatory stimuli cannot be correlated. In this context, one is forced to acknowledge that, say, extrapolations from studies of mouse corneas to the immunological privilege of the cornea (p. 996 in Ambati et al. (2006)) or results from corneal angiogenesis induced by acute inflammatory stimuli (Figure 5 in Cursiefen et al. (2006a)) may have been entirely appropriate at the time but, in light of newly published evidence, represent areas where additional research is needed to solidify the experimental support for such general statements.

Aside from the above-discussed evidence, the issue of corneal avascularityalbeit in a different model organism, Chakravarti et al. (2004) provided support for the notion that murine corneal keratinocytes of the stroma may assume functions of monocytes-derived macrophages, based on microarray studies of cultured stromal cells. While far from formal proof that keratinocytes may commit to a monocyte-lineage development, this suggestion ties in nicely with a report suggesting that inflammation-elicited lymphangiogenesis 
in mouse corneal transplantation assays derives from innate immunity CD11b+CD11c-macrophages (Maruyama et al., 2005). These macrophages then play an active role in establishing the lymphatic vasculature by functional de-novo surface decoration with LYVE-1 and forming the onsets of vascular sheaths (Maruyama et al., 2005). If this is indeed the case and holds true for other organisms than the mouse, this would tie in nicely with the observation that developing human corneas, free from irritation, appear to be free of LYVE-1-positive lymphatic vessels (Cursiefen et al., $2006 \mathrm{~b}$ ). The broader implication of the de-novo surface decoration of innate immune macrophages in the irritated mouse cornea with markers of lymphatic vessels could be, if true for man, that further research efforts may shed light onto the cell biology of the lingering enigma of corneal vascularization in response to irritation.

\section{Acknowledgement}

Joachim Storsberg and Christian Schmidt gratefully acknowledge support from the Fraunhofer Lighthouse Project Theranostic Implants. Mark A. Brown received financial support from the Short Memorial Endowment and The National Science Foundation (1060548). The content of this paper is entirely the responsibility of the authors and does not necessarily represent the official views of either The Fraunhofer Society for the advancement of applied research or The National Science Foundation.

\section{Author's Contributions}

Saadettin Sel, Mark A. Brown and Joachim Storsberg: Provided critical input and assisted in revising and improving the paper.

Christian Schmidt: Wrote the first draft of the paper and provided critical input and assisted in revising and improving the paper.

\section{Ethics}

Saadettin Sel, Mark A. Brown, Joachim Storsberg and Christian Schmidt report no conflicts of interest with regard to this report. Mark A. Brown and Christian Schmidt are members of the Editorial Board of The American Journal of Immunology, are waived from the Article Processing fee for this contribution and receive no remuneration for the editorial work, either individually or collectively.

\section{Other Remarks}

Limiting the scope of this paper forced us to select citations; the references chosen are not seen as superior to the references available forcitation. As a result, we, the authors, wholeheartedly apologize to all colleagues whose importantstudies were not cited.

\section{References}

Ambati, B.K., M. Nozaki, N. Singh, A. Takeda and P. Jani et al., 2006. Corneal avascularity is due to soluble VEGF receptor-1. Nature, 443: 993-997. DOI: 10.1038 /nature05249

Baschuk, N., J. Rautela and B.S. Parker, 2015. Bone specific immunity and its impact on metastasis. Bonekey Rep., 4: 665-665. DOI: $10.1038 /$ bonekey.2015.32

Burnet, M., 1957. Cancer-a biological approach. Br. Med. J., 1: 779-786. DOI: 10.1136/bmj.1.5022.779

Campbell, F.W. and I.C. Michaelson, 1949. Bloodvessel formation in the cornea. Br. J. Ophthalmol., 33: 248-255. DOI: 10.1136/bjo.33.4.248

Chakravarti, S., F. Wu, N. Vij, L. Roberts and S. Joyce, 2004. Microarray studies reveal macrophage-like function of stromal keratocytes in the cornea. Invest. Ophthalmol. Vis. Sci., 45: 3475-3484.

DOI: $10.1167 /$ iovs.04-0343

Cursiefen, C., L. Chen, M. Saint-Geniez, P. Hamrah, Y. Jin and S. Rashid et al., 2006a. Nonvascular VEGF receptor 3 expression by corneal epithelium maintains avascularity and vision. Proc. Natl. Acad., Sci. USA, 103: 11405-11410. DOI: 10.1073/pnas.0506112103

Cursiefen, C., C. Rummelt, A. Jünemann, C. Vorwerk and W. Neuhuber et al., 2006b. Absence of blood and lymphatic vessels in the developing human cornea. Cornea, 25: 722-726. DOI: 10.1097/01.ico.0000214230.21238.3d

Davis, M.M., 2008. A prescription for human immunology. Immunity, 29: 835-838. DOI: 10.1016/j.immuni.2008.12.003

Griffith, M. and D.G. Harkin, 2014. Recent advances in the design of artificial corneas. Curr. Opin. Ophthalmol., 25: 240-247. DOI: $10.1097 /$ ICU.0000000000000049

Halsted, W.S., 1913. Ligature and suture material the employment of fine silk in preference to catgut and the advantages of transfixion of tissues and vessels in control of hemorrhage also an account of the introduction of gloves, gutta-percha tissue and silver foil. JAMA, 60: 1119-1126. DOI: $10.1001 /$ jama.1913.04340150001001

Jordan, T., I. Hanson, D. Zaletayev, S. Hodgson and J. Prosser et al., 1992. The human PAX6 gene is mutated in two patients with aniridia. Nat. Genet., 1: 328-332. DOI: $10.1038 / \mathrm{ng} 0892-328$

Kendall, R.L., G. Wang and K.A. Thomas, 1996. Identification of a natural soluble form of the vascular endothelial growth factor receptor, FLT-1 and its heterodimerization with KDR. Biochem. Biophys. Res. Commun., 226: 324-328.

DOI: $10.1006 /$ bbrc. 1996.1355

Lightbody, W.H., 1867. Observations on the comparative microscopic anatomy of the cornea of vertebrates. J. Anat. Physiol., 1: 15-43. 
Maruyama, K., M. Ii, C. Cursiefen, D.G. Jackson and H. Keino et al., 2005. Inflammation-induced lymphangiogenesis in the cornea arises from CD11b-positive macrophages. J. Clin. Invest., 115: 2363-2372. DOI: $10.1172 /$ JCI23874

McPherson, S.D., 1959. The use of absorbable sutures in surgery of the cornea. Trans. Am. Ophthalmol. Soc., 57: 700-737.

Nelson, L.B., G.L. Spaeth, T.S. Nowinski, C.E. Margo and L. Jackson, 1984. Aniridia. A review. Surv. Ophthalmol., 28: 621-642. DOI: 10.1016/0039-6257(84)90184-X

Niederkorn, J.Y. and J. Stein-Streilein, 2010. History and physiology of immune privilege. Ocul. Immunol. Inflamm., 18: 19-23. DOI: $10.3109 / 09273940903564766$

Ramaesh, T., J.M. Collinson, K. Ramaesh, M.H. Kaufman and J.D. West et al., 2003. Corneal abnormalities in pax6 $6^{+-}$small eye mice mimic human aniridiarelated keratopathy. Invest. Ophthalmol. Vis. Sci., 44: 1871-1878. DOI: 10.1167/iovs.02-0576
Salthouse, T.N., 1976. Cellular enzyme activity at the polymer-tissue interface: A review. J. Biomed. Mater. Res., 10: 197-229. DOI: 10.1002/jbm.820100204

Salthouse, T.N., G.Z. Kaminska, M.L. Murphy, J.A. Williams and D.A. Willigan, 1970. Suture absorption in rabbit cornea and sclera: Enzyme histochemical and morphologic observations. Invest. Ophthalmol., 9: 844-856.

Salthouse, T.N. and B.F. Matlaga, 1975. Significance of cellular enzyme activity at nonabsorbable suture implant sites: Silk, polyester and polypropylene. J. Surg. Res., 19: 127-132. DOI: $10.1016 / 0022-4804(75) 90117-1$

Seok, J., H.S. Warren, A.G. Cuenca, M.N. Mindrinos and H.V. Baker et al., 2013. Genomic responses in mouse models poorly mimic human inflammatory diseases. Proc. Natl. Acad. Sci. USA, 110: 3507-3512. DOI: $10.1073 /$ pnas. 1222878110 